\title{
Texture Determination in Aluminium Alloys using Colour Metallography
}

\author{
S. KRÖGER, E. DAHLEM-KLEIN, H. WEILAND and \\ H. J. BUNGE
}

Department of Physical Metallurgy, Technical University of Clausthal, FRG

(Received September 4, 1987)

Texture components in aluminium alloys can be distinguished by colour metallography using anodic oxidation and polarization microscopy. This method is particularly useful for texture inspection in correlation with other structural parameters such as grain size, orientation correlation and texture inhomogeneity. The method reveals, however, only two of three orientation parameters. It is therefor not suitable to obtain complete orientation distribution functions.

KEY WORDS: Polarization microscopy, Electron diffraction, Kikuchi diagrams, Colour photography, ODF analysis.

\section{INTRODUCTION}

Texture determination can be based on pole figure measurements (taken by x-rays, neutron or electron diffraction) or on individual orientation measurements. Pole figure measurements are considered to be the "normal" method of texture determination. Single orientation measurements are usually more time consuming since a rather large number of crystallites must be measured in order to obtain a statistically relevant orientation distribution function. Furthermore, the determination of the orientation of an individual grain requires substantial manual work and is difficult to 
automate. On the other hand, there are cases where it is really necessary to take resort to individual orientation measurements. This is always the case when the texture of selected groups of crystallites is to be determined. Examples of such cases are textures of individual phases in polyphase materials, especially if the considered phase is present only in a small volume fraction or if its Bragg-angles are systematically superposed with those of other phases of the material. Other examples are textures of grain size classes, e.g. the textures of large or small grains during grain growth or the orientation distribution of grains having a specific environment e.g. those having large angle or small angle grain boundaries, i.e. having a specific orientation correlation with respect to their neighbours. And finally, the inhomogeneous distribution of certain texture components in the polycrystalline structure may be an example. In all these cases it is desirable to have an easy method how to determine the crystallographic orientation of individual grains in order to carry out a fast texture determination of the considered group of crystallites.

In aluminium and aluminium alloys, surface layers can be produced by anodic oxidation. It has also long been known that these layers are double refracting such that different grains appear by different grey shades or by different colours in the polarization microscope. The different shades or colours produced by the oxide layer are related to the crystal orientation of the substrate crystal. This effect was studied in detail by Solberg (1986) and by Saetre, Solberg and Ryum (1986) who pointed out that the surface layer is biaxially double refracting with the axes of double refraction strictly related to the orientation of the aluminium crystal on which it is grown. Hence, two of the three orientation angles of the grain can be determined by measuring the position of maximum and minimum reflected intensity during rotation of the sample under the microscope as well as the amplitude of maximum intensity. The optical path difference of ordinary and extraordinary beam obtained by the light beam passing the oxide layer twice is rather small compared with wavelength. Hence, the colours seen in the polarization microscope are rather pale. They can, however, be enhanced by using a $\lambda / 4$ plate. This way strong colours can be obtained which can easily be distinguished on a colour photograph and which are related to the orientation of the crystallite. As was 
shown by Saetre et al., the accuracy of the determination of the two orientation angles is in the order of a few degrees. There remains, however, one degree of freedom since crystal orientation is determined by three orientation parameters. Hence, it must be possible by optical methods to distinguish groups of crystal orientations having the same two orientation parameters (These groups are integrals of the orientation distribution function ODF in the orientation space along a one-dimensional path).

\section{Experimental procedure}

In the present investigation an AlMn1 alloy was studied. Its primary recrystallization texture was a cube texture with rather large scattering. During continuous grain growth the material developed other textures consisting of several components. Orientation determination was carried out in a $300 \mathrm{KV}$ transmission electron microscope. For these measurements, the samples were electrolytically thinned using an electrolyte consisting of $100 \mathrm{ml}$ perchloric acid, $200 \mathrm{ml}$ glycerine, $700 \mathrm{ml}$ methanol at $10^{\circ} \mathrm{C}$ and a voltage of $10 \mathrm{~V}$. Thereafter the samples were anodized using the electrolyte according to Barker (1950) consisting of $\mathrm{HBF}_{4}+$ $\mathrm{H}_{2} \mathrm{O}, 3: 80$, a current density of $0.2 \mathrm{~A} / \mathrm{cm}^{2}$ at $20^{\circ} \mathrm{C}$ and anodizing times between 20 and 120 seconds. The samples were than studied in a polarization microscope with crossed polarizers using a $\lambda / 4$ plate and the rolling direction being parallel to the polarization direction. These conditions were kept fixed for all measurement. Colour photographs were taken in order to evaluate the colour of each grain. Transparent foils were investigated by electron diffraction. The anodic oxide layer was transparent for $300 \mathrm{KV}$ electrons and no diffraction Pattern was obtained from the layer. The orientation of the aluminium crystals was studied using Kikuchi patterns, which were evaluated semiautomatically in the microscope. In order to measure the positions of at least three Kikuchi bands, four points on each band were shifted subsequently into the origin using two pairs of deflection coils of the microscope. The voltage across the coils which is a meausre of the $x-y$ coordinates of each point can be read into the controlling computer by an AD-converter as is shown in Figure 1. The orientation of the grain is then calculated by an appropriate computer program (Weiland 

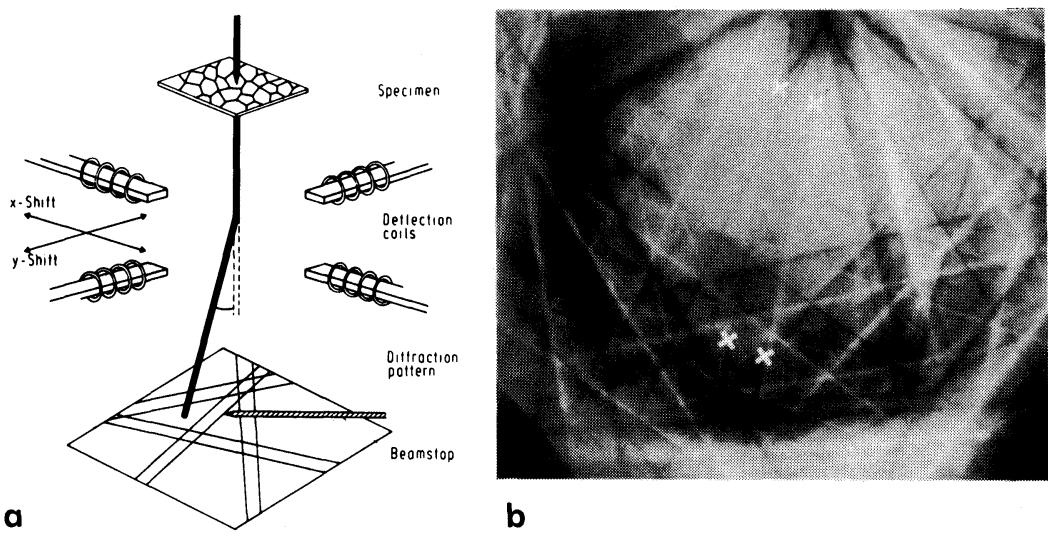

Figure 1 Orientation determination in the TEM by Kikuchi diagrams. (a) Measurement of $x-y$ coordinates by image deflection; (b) Kikuchi pattern with four points characterizing a Kikuchi band.

and Schwarzer 1986). For the following calculation of orientation distribution functions the orientation is expressed in terms of Euler angles $\phi_{1}, \emptyset, \phi_{2}$. The accuracy of this procedure is about $1^{\circ}$. Orientation distribution functions are then calculated using the series expansion method

$$
f(g)=\sum_{l=0}^{L} \sum_{\mu=1}^{M(l)} \sum_{v=1}^{N(l)} C_{l}^{\mu \nu}, T_{l}^{\mu \nu}(g) ; \quad g=\left(\phi_{1}, \emptyset, \phi_{2}\right)
$$

with the coefficients

$$
C_{l}^{\mu \nu}=\alpha(l) \frac{\sum_{i} w_{i} T_{l}^{\mu \nu}\left(g_{i}\right)}{\sum_{i} w_{i}}
$$

where $g_{i}$ are the orientations of the individual grains and $w_{i}$ are weight factors which may be chosen as unity or proportional to the area of the grain. The factor $a(1)$ is a convergence or smoothing factor which is choosen according to the number of measured orientations. It is

$$
\alpha(l)=\frac{\exp \left(-\frac{1}{4} l^{2} \omega^{2}\right)-\exp \left(-\frac{1}{4}(l+1)^{2} \omega^{2}\right)}{1-\exp \left(-\frac{1}{4} \omega^{2}\right)}
$$

In the present case the "smoothing angle" angle $\omega$ was chosen $\omega=8^{\circ}$. The degree of series expansion was $L=22$. 


\section{RESULTS}

In a preliminary investigation the reproducibility of colours was investigated. A sample was anodized, and a photograph was taken. Then the layer was removed by polishing and the sample was again anodized using the same conditions (as best they could be reproduced). The colour photographs of the same group of grains obtained after the first and second anodization are shown in Figure 2. With respect to the rather rough colour clasification to be used in the following the colours were sufficiently reproduced. In a second preliminary investigation the influence of the anodization time was tested. A sample was anodized $20 \mathrm{sec}$ and a photograph was taken. Then the anodization was continued and so on till to a total anodization time of $210 \mathrm{sec}$. The so obtained colours of the same grains are shown in Figure 3 . It is seen that the colours are slightly changing but the character remains constant. The samples of Figures 2 and 3 belonged to different texture types. Besides this, the photographs were taken with different illumination in Figures 2

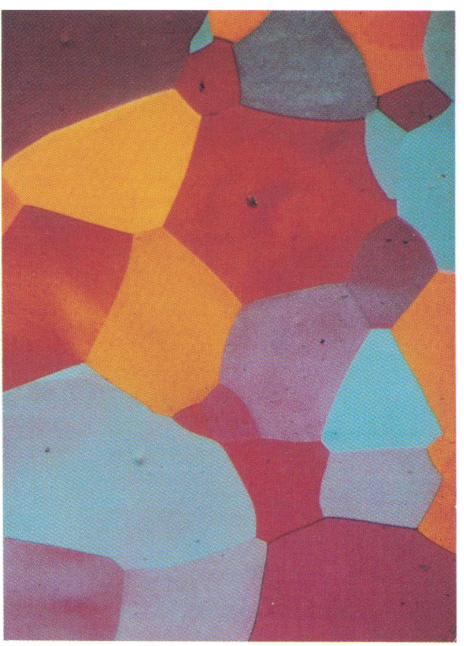

a

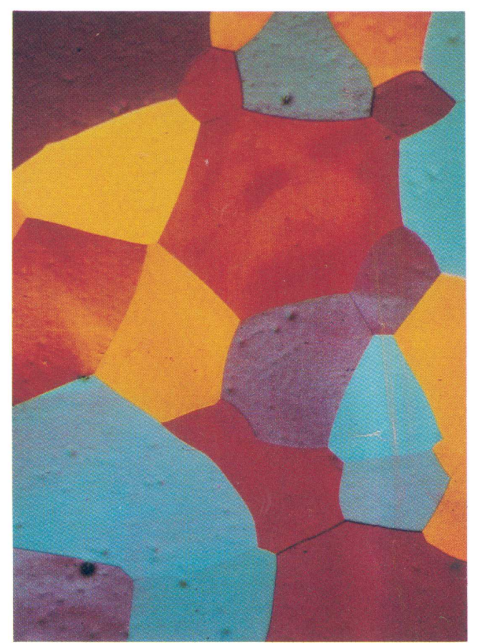

b

Figure 2 Repeated anodizing of the same sample reveals nearly the same colours. (a) first preparation; (b) second preparation. (See Color Plates I and II.) 


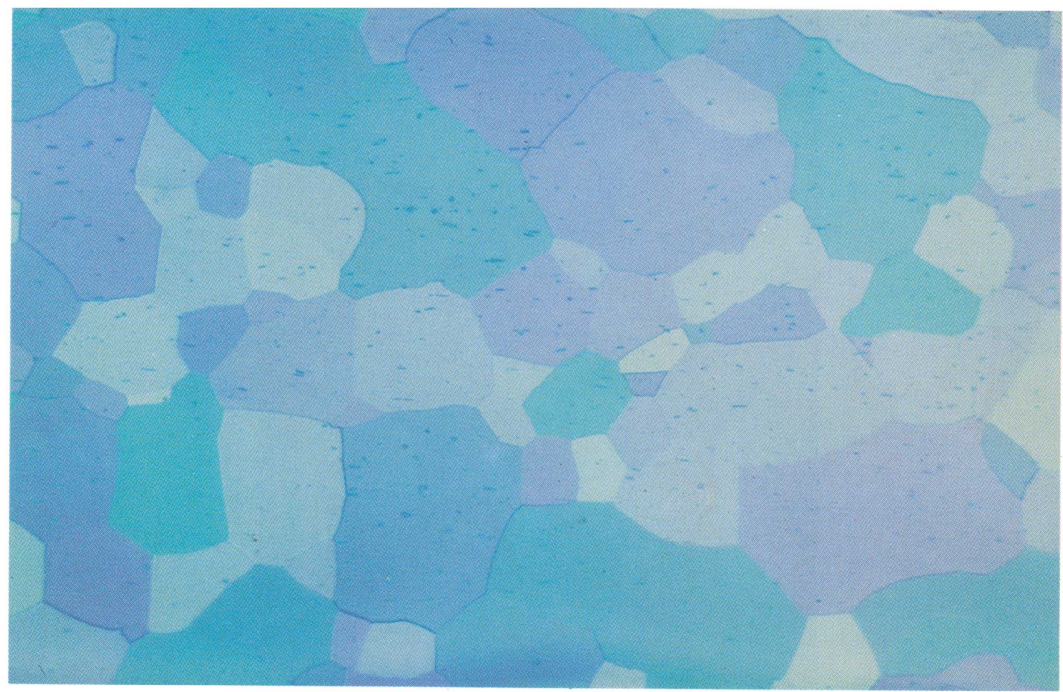

a

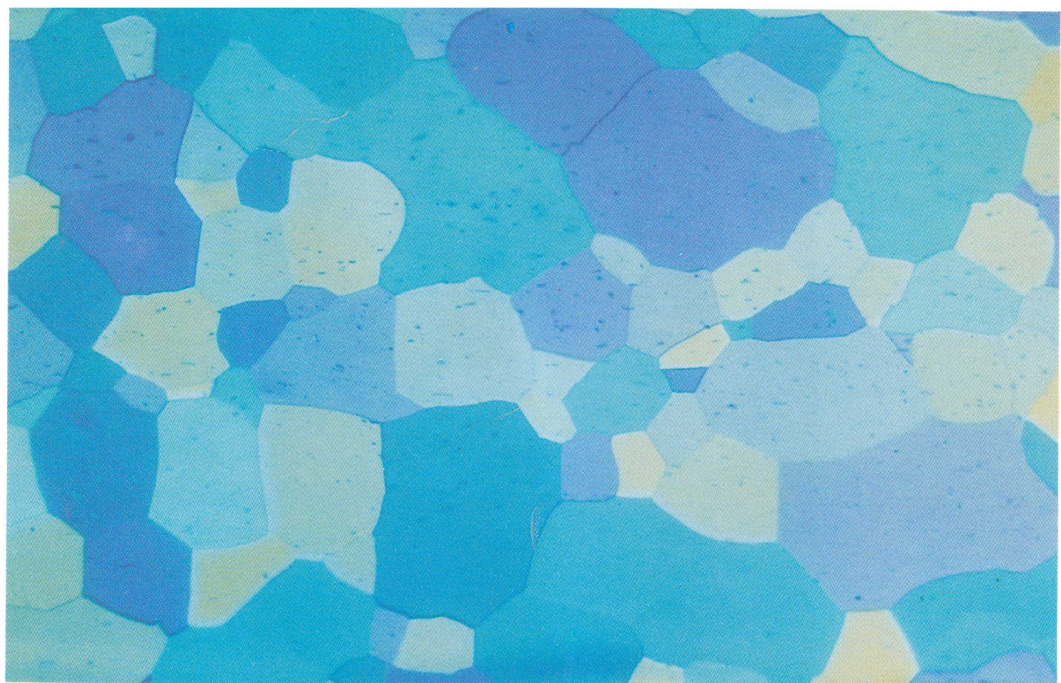

b

Figure 3 Continued oxidizing from $20 \mathrm{sec}$ to $210 \mathrm{sec}$. (a) $20 \mathrm{sec}$ oxidation time; (b) $210 \mathrm{sec}$ oxidation time. (See Color Plates III and IV.) 


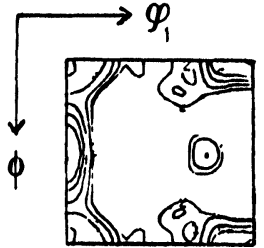

a

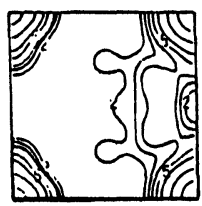

C

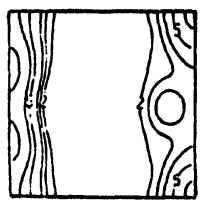

b

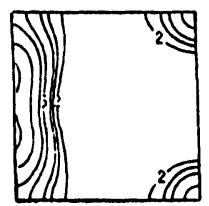

d

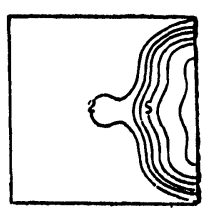

e

Figure 4 Orientation distribution functions of an AlMn1 sample after cold rolling, recrystallization and grain growth $\left(\phi_{2}=0^{\circ}\right.$ section). (a) $\mathrm{x}$-ray diffraction; (b) electron diffraction (all grains); (c) blue grains; (d) yellow grains; (e) red grains.

and 3 respectively. This explains the different overall colours of these two figures.

In order to study the textures of various colour groups the orientations of 114 grains in a sample were measured along with their corresponding colours. The orientation distribution function calculated according to Eqs. 1-3 (with $w_{i}=1$ ) is shown in Figure 4b. It agrees quite well with that obtained by $x$-ray diffraction and pole figure inversion Figure $4 \mathrm{a}$, bearing in mind the different weight factors used in both methods. With x-ray diffraction it is $w_{i} \approx v_{i}\left(v_{i}\right.$ being the volume of the grain) as compared with $w_{i}=1$ used in electron diffraction (ODF by volume and ODF by number respectively). (See e.g. Dahlem-Klein et al. 1987) The ODF's were then calculated for each colour group separately as is also shown in Figure 4c-e.

\section{Conclusions}

As was to be concluded from the results by Saetre, Solberg and Ryum (1986), groups of texture components can be distinguished in 
aluminium alloys using colour microscopy. This is confirmed by the present results. Rather rough colour classification already reveals different texture components as is seen in Figure 4. Hence, the method is suitable to obtain a fast survey on texture components. It is particulary suited in cases where the texture is, in principle, known and contains only a limited number of components. Colour classification of the texture components may then be used to relate crystal orientation or orientation correlation to other spacial parameters of the polycrystalline structure such as grain size orientation correlation or texture inhomogeneties. Since the method, as shown above, can only distinguish two of the three orientation parameters it is not suitable for complete texture determinations.

\section{References}

Barker L. J. (1950) Trans ASM 42, 347-356.

Dahlem-Klein, E., Weiland, H., Fiszer, A. and Bunge, H. J., Scripta Met. submitted for publication.

Saetre T. O., Solberg J. K. and Ryum M. (1986) Metallography 19, 345-357.

Solberg J. K., Metallography (1986) 19, 197-207.

Weiland H. and Schwarzer R. (1986) In: Experimental Techniques of Texture Analysis Ed. H. J. Bunge. DGM Informationsgesellschaft Oberursel pp. 301-313. 